\title{
N-enriched GO Adsorbent Series for Selective Adsorption of CO2: Characterization, Equilibrium, and Thermodynamic Studies
}

\author{
Mahsa Najafi ${ }^{1}$, Yasamin Hosseini ${ }^{2}$, Soodabeh Khalili ${ }^{3}$, Majid Peyravi ${ }^{2}$, and Mohsen \\ Jahanshahi $^{2}$ \\ ${ }^{1}$ University of Tehran College of Engineering \\ ${ }^{2}$ Affiliation not available \\ ${ }^{3}$ Babol Noshirvani University of Technology
}

April 10, 2021

\begin{abstract}
In this study a series of GO-based adsorbents were assembled via impregnation method using N-resources: 3-aminopropyltriethoxysilane (APTS) as primary amio-silane, Piperazine (PIP) as secondary cyclic diamine, and ethanolamine (EA) as primary amine. The influence of amine type, adsorption temperature and pressure were undertaken to obtain the best CO2 adsorption performance. The characterizing techniques including FTIR, SEM, TGA, BET, BJH, and MP confirmed well impregnation of amine functionalities to the GO framework and high thermal stability of adsorbents. GO/APTS showed the maximum CO2 uptake $(43.114 \mathrm{mmol} / \mathrm{g})$ predicted by the Sips isotherm model and the highest CO2 $\neg(15 \% \mathrm{~V}$, balanced N2) selectivity $(33.7 \%$ ) estimated by the ideal adsorbed solution theory. The experimental adsorption capacity of GO/APTS is 2.3 times higher than pristine GO. This behavior highlights the role of electron-donor amine and methyl groups and high molecular weight of APTS as well as high interfacial area of GO/APTS in CO2 capture.
\end{abstract}

\section{Hosted file}

MANUSCRIPT--.pdf available at https://authorea.com/users/407042/articles/517474-n-enrichedgo-adsorbent-series-for-selective-adsorption-of-co2-characterization-equilibrium-andthermodynamic-studies 